\title{
Hyperasymptotics and the Stokes' phenomenon
}

\author{
A. B. Olde Daalhuis \\ CWI, P.O. Box 4079, 1009 AB Amsterdam, The Netherlands \\ email: aod@cwi.nl
}

(MS received 29 April 1992. Revised MS received 24 June 1992)

\begin{abstract}
Synopsis
Hyperasymptotic expansions were recently introduced by Berry and Howls, and yield refined information by expanding remainders in asymptotic expansions. In a recent paper of Olde Daalhuis, a method was given for obtaining hyperasymptotic expansions of integrals that represent the confluent hypergeometric $U$-function. This paper gives an extension of that method to neighbourhoods of the so-called Stokes lines. At each level, the remainder is exponentially small compared with the previous remainders. Two numerical illustrations confirm these exponential improvements.
\end{abstract}

\section{Introduction}

In this paper, we give hyperasymptotic expansions for the confluent hypergeometric functions $U(a, c, z)$ for large $|z|$, where $z$ is in a neighbourhood of a so-called Stokes line. An integral representation for the $U(a, c, z)$ is

$$
U(a, c, z)=\frac{1}{\Gamma(a)} \int_{0}^{\infty} e^{-z t t^{a-1}}(1+t)^{-b} d t,
$$

where $b=a-c+1$ and where the constant $a$ fulfils $: i a>0$.

First we give a general description of hyperasymptotic expansions. Simple asymptotic expansions are of the form $f(z)=a_{0}+a_{1} z^{-1}+\ldots+a_{N_{0}-1} z^{1-N_{0}}+$ $R_{N_{0}}(z)$, where $R_{N_{0}}(z)=\mathscr{O}\left(z^{-N_{10}}\right)$ as $|z| \rightarrow \infty$. The optimal $N_{0}$ can be obtained by minimisation of the remainder $R_{N_{0}}$ when $z$ is given. Generally, $N_{0}$ is a function of $z$. Considering $R_{N_{0}}$ as a function depending on two large parameters ( $z$ and $N_{0}$ ), it is expanded in a new asymptotic expansion, which is truncated at $N_{1}: R_{N_{0}}(z)=B_{0}+B_{1}+\ldots+B_{N_{1}-1}+R_{N_{1}}(z)$, where the remainder $R_{N_{1}}(z)$ appears to be exponentially small compared with $R_{N_{i}}(z)$, as $|z| \rightarrow \infty$. The $B_{m}$ are not of the form const. $z^{-M-m}$. Repeating this, we obtain

$$
f(z)=a_{0}+\ldots+a_{N_{0}-1} z^{1-N_{0}}+B_{0}+\ldots+B_{N_{1}-1}+C_{0}+C_{1}+\ldots+R_{N_{n}}(z)
$$

where $R_{N_{n}}$ appears to be exponentially small compared with $R_{N_{n-1}}$, i.e. $R_{N_{n}}=\mathcal{O}\left(e^{-\lambda_{n}|z|}\right)$, as $|z| \rightarrow \infty$, where $0<\lambda_{0}<\lambda_{1}<\lambda_{2}<\ldots$

A hyperasymptotic expansion for $U(a, c, z)$ is given in [5], for $|z| \rightarrow \infty$. This expansion is limited to the sector $|\arg (z)|<\pi$. This is due to new exponentially small terms in the asymptotic expansion of $U(a, c, z)$, which appear in neighbourhoods of the Stokes lines $\arg (z)= \pm \pi$. These new terms are of the same order as 
$R_{N_{0}}(z)$. For hyperasymptotic expansions in a neighbourhood of these Stokes lines, we need more complicated functions than the functions used in [5].

Two asymptotic expansions for $U(a, c, z)$, in a neighbourhood of $\arg (z)=\pi$, are

$$
\begin{gathered}
U(a, c, z) \sim z^{-a} \sum_{n=0}^{\infty}(-1)^{n} \frac{(a)_{n}(b)_{n}}{n ! z^{n}}, \quad|\arg (z)| \leqq \frac{3}{2} \pi-\delta, \\
U(a, c, z) \sim z^{-a} \sum_{n=0}^{\infty}(-1)^{n} \frac{(a)_{n}(b)_{n}}{n ! z^{n}} \\
-2 \pi i \frac{e^{-(a+b) \pi i}}{\Gamma(a) \Gamma(b)} z^{b-1} e^{z} \sum_{n=0}^{\infty} \frac{(1-a)_{n}(1-b)_{n}}{n ! z^{n}}, \quad \frac{1}{2} \pi-\delta \leqq \arg (z) \leqq \frac{5}{2} \pi+\delta,
\end{gathered}
$$

where Pochhammer's symbol $(a)_{n}$ is defined by $(a)_{n}=\Gamma(a+n) / \Gamma(a)$, and where $\delta$ is a small positive constant. These expansions are combined into the following expansion given in [8]:

$$
\begin{aligned}
U(a, c, z)= & z^{-a} \sum_{n=0}^{N-1}(-1)^{n} \frac{(a)_{n}(b)_{n}}{n ! z^{n}}+R_{N}(z), \\
R_{N}(z)= & (-1)^{N} 2 \pi \frac{z^{b-1} e^{z}}{\Gamma(a) \Gamma(b)} \sum_{m=0}^{M-1}(-1)^{M} \frac{(1-a)_{m}(1-b)_{m}}{m !} \\
& \times \frac{F_{N-m+a+b-1}(z)}{z^{m}}+R_{M}(z),
\end{aligned}
$$

where $N=|z|-a-b+1+\alpha,|\alpha|$ being bounded, and where $M$ is an arbitrary fixed integer. This expansion holds for $|\arg (z)| \leqq \frac{5}{2} \pi-\delta$. The extra functions that we shall use in hyperasymptotic expansions of $U(a, c, z)$, in neighbourhoods of the Stokes lines, are

$$
F_{p}(z)=\frac{e^{-z}}{2 \pi} \int_{0}^{\infty} \frac{e^{-z t} t^{p-1}}{1+t} d t
$$

This function is closely related to one of the incomplete gamma functions in the following way: $F_{p}(z)=(2 \pi)^{-1} \Gamma(p) \Gamma(1-p, z)$. In [7] it is shown that

$$
F_{N+p}(z)=\frac{1}{2}(-1)^{N} i e^{-p \pi i} \operatorname{erfc}\{c(\theta) \sqrt{\rho}\}+\mathcal{O}\left(\left|\frac{\exp \left(-\rho[c(\theta)]^{2}\right)}{\sqrt{\rho}}\right|\right), \quad \text { as } \quad|z| \rightarrow \infty,
$$

where $z$ is written as $z=\rho e^{i \theta}$, and where $p$ is fixed and $|N-\rho|$ is bounded; furthermore $c(\theta)=\sqrt{e^{i \theta}+1+i(\theta-\pi)}$. This expansion combined with (1.5) shows the appearance of new exponentially small terms in the asymptotic expansion of $U(a, c, z)$, in a neighbourhood of the Stokes lines $\theta= \pm \pi$. 
The hyperasymptotic expansion presented in this paper is of the form

$$
\begin{aligned}
U(a, c, z)= & z^{-a} \sum_{n=0}^{N_{0}-1}(-1)^{n} \frac{(a)_{n}(b)_{n}}{n ! z^{n}} \\
& +(-1)^{N_{0}} 2 \pi \frac{e^{z}}{\Gamma(a) \Gamma(b)} U\left(1-b, c, \rho e^{i(\theta-\pi)}\right) F_{a+N_{0}}(z) \text { as } \rho \rightarrow \infty \\
& + \text { N.H.E. }
\end{aligned}
$$

and it holds for $\delta \leqq \theta \leqq 2 \pi-\delta$, where, again, $z=\rho e^{i \theta}$ and $\delta$ is a small positive constant. The N.H.E. stands for Normal Hyperasymptotic Expansion, which is of the form described at the beginning of this section and in [5].

\section{Hyperasymptotic expansions of $U(a, c, z)$, for $|\arg (z)|<\pi$}

In this section we summarise the results and the method of [5]. We write $z=\rho e^{i \theta}$ and we take $t=\tau e^{-i \theta}$, where $-\pi+\delta \leqq \theta \leqq \pi-\delta$ and $\delta$ small and positive. In this way we obtain an analytic continuation with respect to this $z$-domain for $U(a, c, z)$, with

$$
U(a, c, z)=\frac{e^{-i a \theta}}{\Gamma(a)} \int_{0}^{\infty} e^{-\rho \tau} \tau^{a-1}\left(1+\tau e^{-i \theta}\right)^{-b} d \tau
$$

We define $f_{0}(\tau)=\left(1+\tau e^{-i \theta}\right)^{-b}$ and we expand $f_{0}(\tau)$ at $\tau=0$

$$
f_{0}(\tau)=\sum_{n=0}^{N_{0}-1} \frac{(b)_{n}}{n !}\left(-\tau e^{-i \theta}\right)^{n}+\tau^{N_{0}} f_{1}(\tau)
$$

Taylor's theorem gives for $f_{1}(\tau)$ the following integral representation:

$$
f_{1}(\tau)=\frac{1}{2 \pi i} \int_{\Omega_{1}(0, \tau)} \frac{f_{0}(w) d w}{w^{N_{0}}(w-\tau)},
$$

where we choose $\Omega_{0}(0, \tau)$ the union of the circles $|w|=1-\varepsilon$ and $|w-\tau|=\varepsilon / 2$, where $0<\varepsilon<\frac{1}{2}$ and $\varepsilon$ is bounded away from 0 . In the case that $|\tau| \leqq 1-\varepsilon / 2$, we have to adjust this contour. See Figure 2.1. On $\left|\tau+e^{i \theta}\right| \geqq \varepsilon$ we have $\left|f_{0}(\tau)\right| \leqq \varepsilon^{-: i b}$,
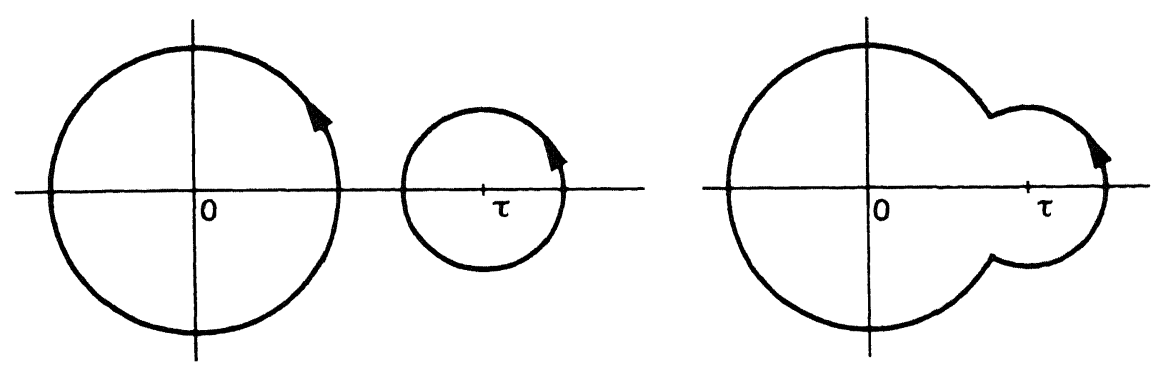

Figure 2.1. The normal and adjusted contour of integration of (2.3). 
and with (2.3) we obtain

$$
\left|f_{1}(\tau)\right| \leqq C_{1}(1-\varepsilon)^{-N_{0}}, \quad \text { as } \quad\left|\tau+e^{i \theta}\right| \geqq \frac{3}{2} \varepsilon,
$$

where $C_{1}$ does not depend on $N_{0}$. We substitute (2.2) in (2.1) and obtain

$$
U(a, c, z)=z^{-a} \sum_{n=0}^{N_{0}-1}(-1)^{n} \frac{(a)_{n}(b)_{n}}{n ! z^{n}}+R_{N_{0}}(z),
$$

with

$$
R_{N_{0}}(z)=\frac{e^{-i a \theta}}{\Gamma(a)} \int_{0}^{\infty} e^{-\rho \tau} \tau^{a-1+N_{0}} f_{1}(\tau) d \tau .
$$

With (2.4) we estimate

$$
\left|R_{N_{0}}(z)\right| \leqq \frac{C_{1}}{|\Gamma(a)|} \int_{0}^{\infty} e^{-\rho \tau} \tau^{\Im: a-1+N_{0}}(1-\varepsilon)^{-N_{0}} d \tau .
$$

The integrand has a maximum at $\tau=\gamma_{1}$, where $\gamma_{1}$ fulfils $\rho=\left(\Re a-1+N_{0}\right) / \gamma_{1}$. This maximum is $e^{-\rho \gamma_{1}} \gamma_{1}^{\rho \gamma_{1}}(1-\varepsilon)^{\Re i a-1-\rho \gamma_{1}}$, and it is the main factor in asymptotic estimations of the right-hand side of (2.7). This factor as a function of $\gamma_{1}$ is minimal at $\gamma_{1}=1-\varepsilon$. So, we choose $N_{0}=[\rho(1-\varepsilon)-\Re a+1]$ and afterwards we take $\gamma_{1}=\left(\Re a-1+N_{0}\right) / \rho$, where $[y]$ denotes the integer part of $y$. This $z$-dependence of $N_{0}$ is typical in hyperasymptotic expansions, and with this $N_{0}$ the remainder is minimal. Laplace's method (see [6, p. 80]) gives the estimate

$$
\left|R_{N_{0}}(z)\right|=\mathcal{O}\left(C_{1} \sqrt{\frac{2 \pi}{\rho}} e^{-\rho \gamma_{1}} \gamma_{1}^{i j a-\frac{1}{2}}\right), \quad \text { as } \quad|z| \rightarrow \infty .
$$

Now we describe the method for obtaining the $(n+1)$ th-level of the hyperasymptotic expansion from the remainder of the $n$ th-level. More details are given in [5], and the next section is more detailed. The remainder at the $n$ th-level is of the form

$$
R_{N_{n}}(z)=\frac{e^{-i a \theta}}{\Gamma(a)} \int_{0}^{\infty} e^{-\rho \tau} \tau^{a-1+N_{0}}\left(\tau-\gamma_{1}\right)^{N_{1}} \ldots\left(\tau-\gamma_{n}\right)^{N_{n}} f_{n+1}(\tau) d \tau,
$$

where

$$
f_{n+1}(\tau)=\frac{1}{2 \pi i} \int_{\Omega_{n}\left(\gamma_{n}, \tau\right)} \frac{f_{n}(w) d w}{\left(w-\gamma_{n}\right)^{N_{n}}(w-\tau)},
$$

and where $\Omega_{n}\left(\gamma_{n}, \tau\right)$ is a contour that encircles $\gamma_{n}$ and $\tau$. These contours look like those in Figure 2.1. The estimations of the $R_{N_{n}}$ are of the form

$$
\left|R_{N_{n}}(z)\right| \leqq C_{n} \int_{0}^{\infty} e^{-\rho \tau} \frac{\tau^{\Re i a-1+N_{0}}}{(1-\varepsilon)^{N_{0}}}\left|\frac{\tau-\gamma_{1}}{d\left(\gamma_{1}, \theta\right)-\rho_{1} \varepsilon}\right|^{N_{1}} \cdots\left|\frac{\tau-\gamma_{n}}{d\left(\gamma_{n}, \theta\right)-\rho_{n} \varepsilon}\right|^{N_{n}} d \tau,
$$

where $d\left(\gamma_{m}, \theta\right)=\sqrt{\gamma_{m}^{2}+2 \gamma_{m} \cos \theta+1}$ and $\rho_{m}=2-2^{-m}$. To obtain $N_{n}$, such that 


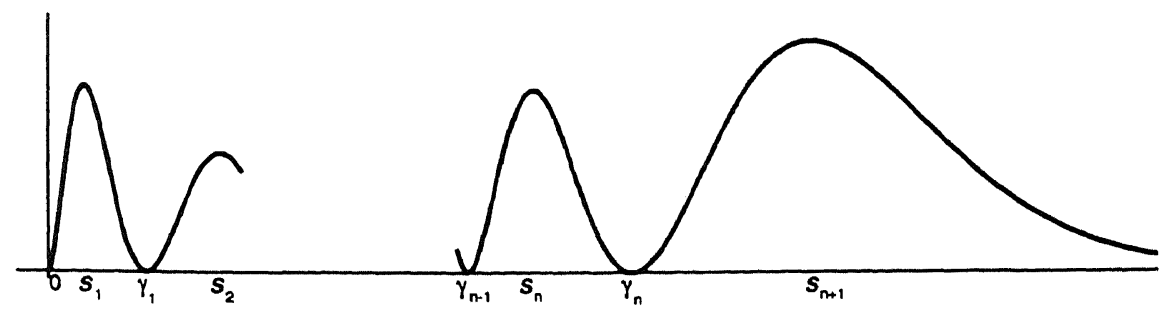

Figure 2.2. The graph of the integrand of (2.11).

the right-hand side of (2.11) is minimal, we analyse the integrand of (2.11). This integrand is of the form shown in Figure 2.2. The local maxima are at $\tau=s_{j}, j=1,2, \ldots, n, n+1$, and these $s_{j}$ fulfil

$$
\rho=\frac{\Re a-1+N_{0}}{s_{j}}+\frac{N_{1}}{s_{j}-\gamma_{1}}+\ldots+\frac{N_{n}}{s_{j}-\gamma_{n}} .
$$

From (2.12) it follows that $s_{1}, \ldots, s_{n}$ are decreasing functions of $N_{n}$, and that $s_{n+1}$ is an increasing function of $N_{n}$. It is not difficult to prove that the local maxima at $s_{1}, \ldots, s_{n}$ are decreasing functions of $N_{n}$, and that the local maximum at $s_{n+1}$ is decreasing on the interval $s_{n+1} \in\left(\gamma_{n}, \gamma_{n}+d\left(\gamma_{n}, \theta\right)-\rho_{n} \varepsilon\right)$ and increasing on the interval $s_{n+1} \in\left(\gamma_{n}+d\left(\gamma_{n}, \theta\right)-\rho_{n} \varepsilon, \infty\right)$. Thus for some $N_{n} \in \mathbb{N}$ the global maximum is minimal, and it is attained at $s_{n+1}$. Now we expand $f_{n+1}(\tau)$ at $\gamma_{n+1}=s_{n+1}$ :

$$
\begin{aligned}
f_{n+1}(\tau)= & b_{0}+b_{1}\left(\tau-\gamma_{n+1}\right)+\ldots+b_{N_{n+1}}\left(\tau-\gamma_{n+1}\right)^{N_{n+1}-1} \\
& +\left(\tau-\gamma_{n+1}\right)^{N_{n+1}} f_{n+2}(\tau) .
\end{aligned}
$$

We substitute this expansion in (2.9) and we obtain the $(n+1)$ th-level

$$
\begin{aligned}
R_{N_{n}}(z)= & \frac{e^{-i a \theta N_{n+1}-1}}{\Gamma(a)} \sum_{m=0}^{-1} b_{m} \int_{0}^{\infty} e^{-\rho \tau} \tau^{a-1+N_{0}} \ldots\left(\tau-\gamma_{n}\right)^{N_{n}} \\
& \times\left(\tau-\gamma_{n+1}\right)^{m} d \tau+R_{N_{n}, 1}(z) .
\end{aligned}
$$

In [5] it is explained how the integrals in (2.14) can be computed.

At the first and second levels of the hyperasymptotic process, the minimal global maximum is attained only at the furthest right maximum (i.e. at $s_{n+1}$ ). For the higher levels this is not obvious, and in the next section we show what happens if the global maximum is attained at more than 1 of the $s_{j}$.

At each level we obtain $\left|R_{N_{n}}(z)\right|=\mathcal{O}\left(\rho^{-\frac{1}{2}} e^{-\lambda_{n} \rho}\right)$ as $|z| \rightarrow \infty$, where $\lambda_{0} \sim 1<\lambda_{1}<\lambda_{2}<\lambda_{3}<\ldots$. We do not have a proof as to whether or not $\left\{\lambda_{n}\right\}_{n \in \mathbb{N}}$ is an unbounded sequence. For $\theta=0$, we have $\lambda_{1} \sim 1.90$ and $\lambda_{2} \sim 3.59$. Thus at the second level our $\lambda_{2}$ already exceeds the limiting of 2.39 of a different method, which is described at the end of the next section.

\section{Hyperasymptotic expansions of $U(a, c, z)$, near the Stokes line $\arg (z)=\pi$}

In this section we shall obtain a hyperasymptotic expansion for $U\left(a, c, \rho e^{i \theta}\right)$ of the form (1.8), which holds for $\delta \leqq \theta \leqq 2 \pi-\delta$. The expansion will be in terms of the function $F_{a+N_{0}}(z)$, and functions of the form $U\left(1-b, c, \lambda \rho e^{i(\theta-\pi)}\right)$, where $\lambda$ is 
a positive constant. Notice that for confluent hypergeometric functions with the present $z$-domain, we have already derived a hyperasymptotic expansion in Section 2 and [5].

We start with the expansion (2.5), and we use [8] where an integral representation for $R_{N_{0}}(z)$ is given in the form

$$
R_{N_{0}}(z)=(-1)^{N_{0}} \frac{e^{-i \theta\left(a+N_{0}-1\right)}}{\Gamma(a) \Gamma(b)} \int_{0}^{\infty} \frac{U(1-b, c, \rho \tau) e^{-\rho \tau} \tau^{a+N_{0}-1}}{\tau+e^{i \theta}} d \tau .
$$

This integral is defined for $-\pi<\theta<\pi$ and $N_{0}>\max (\Re(-a), \mathfrak{R}(-b))$. Now we sub-

stitute

$U(1-b, c, \rho \tau)=\left\{U(1-b, c, \rho \tau)-U\left(1-b, c, \rho e^{i(\theta-\pi)}\right)\right\}+U\left(1-b, c, \rho e^{i(\theta-\pi)}\right)$

in (3.1) and we obtain

$$
\begin{aligned}
R_{N_{0}}(z)= & (-1)^{N_{0}} \frac{e^{-i \theta\left(a+N_{0}-1\right)}}{\Gamma(a) \Gamma(b)} \int_{0}^{\infty} g_{1}(\tau, z) e^{-\rho \tau} \tau^{a+N_{0}-1} d \tau \\
& +\frac{2 \pi(-1)^{N_{0}}}{\Gamma(a) \Gamma(b)} e^{z} U\left(1-b, c, \rho e^{i(\theta-\pi)}\right) F_{a+N_{0}}(z),
\end{aligned}
$$

where we used the integral representation (1.6) for the second term, and where

$$
g_{1}(t, z)=\frac{U(1-b, c, \rho t)-U\left(1-b, c, \rho e^{i(\theta-\pi)}\right)}{t+e^{i \theta}} .
$$

This function is regular in $t=e^{i(\theta-\pi)}$, thus the right-hand side of (3.2) is an analytic continuation of $R_{N_{0}}(z)$ for $-\pi<\theta<3 \pi$.

In the remaining part of this section, we shall give a hyperasymptotic expansion for the first term of the right-hand side of (3.2), that is

$$
S_{N_{0}}(z)=(-1)^{N_{0}} \frac{e^{-i \theta\left(a+N_{0}-1\right)}}{\Gamma(a) \Gamma(b)} \int_{0}^{\infty} g_{1}(\tau, z) e^{-\rho \tau} \tau^{a+N_{0}-1} d \tau .
$$

In the appendix we shall prove that we can bound $g_{1}(t, z)$ by

$$
\left|g_{1}(t, z)\right| \leqq C_{1}(t, \varepsilon) \rho^{\Re(b-1}, \quad \Re(t)>0,
$$

where $C_{1}(t, \varepsilon)=C_{1} \max \left(|t|^{-|\Re a|-|a t b|}, \varepsilon\right)$, with $\varepsilon$ a small positive constant. With (3.5), we estimate

$$
\left|S_{N_{0}}(z)\right| \leqq C_{1} \rho^{: k b-1} \int_{0}^{\infty} e^{-\rho \tau} \tau^{: i a+N_{0}-1} d \tau,
$$

where $C_{1}$ depends on $a, b$ and $\varepsilon$, and for small $\varepsilon$ we can obtain the estimate $\left|S_{N_{0}}(z)\right|=\mathcal{O}\left(\rho^{: \geqslant b-\frac{3}{2}} e^{-\rho}\right)$, as $\rho \rightarrow \infty$.

For the Taylor series of $g_{1}(t, z)$ at $t=\lambda$, where $\lambda$ is a positive number, we can use the next expression:

$$
\frac{d^{n}}{d t^{n}} g_{1}(t, z)=\frac{1}{t+e^{i \theta}}\left[(-\rho)^{n}(1-b)_{n} U(1-b+n, c+n, \rho t)-n \frac{d^{n-1}}{d t^{n-1}} g_{1}(t, z)\right]
$$


which is proved in the appendix. With the recursion relation

$$
U(\alpha+2, \gamma+2, z)=\frac{\gamma-z}{z(\alpha+1)} U(\alpha+1, \gamma+1, z)+\frac{1}{z(\alpha+1)} U(\alpha, \gamma, z),
$$

which follows from Kummer's equation, it follows that the coefficients of the Taylor series of $g_{1}(t, z)$, at $t=\lambda$, can be computed easily from the "known" $U(1-b, c, \rho \lambda), U(2-b, c+1, \rho \lambda)$ and $U\left(1-b, c, \rho e^{i(\theta-\pi)}\right)$.

Now we expand $g_{1}(\tau, z)$ in a Taylor series at $\tau=\gamma_{1}$, where $\gamma_{1}$ is the maximum of the integrand of (3.6), (see Section 2):

$$
g_{1}(\tau, z)=b_{0,1}+b_{1,1}\left(\tau-\gamma_{1}\right)+\ldots+b_{N_{1}-1,1}\left(\tau-\gamma_{1}\right)^{N_{1}-1}+\left(\tau-\gamma_{1}\right)^{N_{1}} g_{2}(\tau, z),
$$

where

$$
g_{2}(t, z)=\frac{1}{2 \pi i} \int_{\Omega_{1}\left(\gamma_{1}, t\right)} \frac{g_{1}(w, z)}{\left(w-\gamma_{1}\right)^{N_{1}}(w-t)} d w,
$$

and where $\Omega_{1}\left(\gamma_{1}, t\right)$ is a contour that encircles $\gamma_{1}$ and $\tau$. For obtaining sharp estimates for $g_{2}(t, z)$, we choose here and below for $n=1,2,3, \ldots$

$$
\Omega_{n}\left(\gamma_{n}, t\right)=\left\{\begin{array}{llr}
\left\{w \in \mathbb{C}|| w-\gamma_{n} \mid=\gamma_{n}-\rho_{n} \varepsilon \text { or }|w-t|=2^{-n} \varepsilon\right\} & \text { for } & \Re t>\rho_{n} \varepsilon, \\
\left\{w \in \mathbb{C}|| w-\gamma_{n} \mid=\gamma_{n}-\rho_{n} \varepsilon \text { or }|w-t|=\frac{1}{2} \Re t\right\} & \text { for } & 0<\Re t \leqq \rho_{n} \varepsilon,
\end{array}\right.
$$

where $\rho_{n}=2-2^{1-n}$. Thus $\Omega_{n}\left(\gamma_{n}, t\right)$ is the union of two circles. In the case that these discs intersect, we take $\Omega_{n}\left(\gamma_{n}, t\right)$ the outer part of these two circles. (See Figure 2.1.) With this $\Omega_{1}\left(\gamma_{1}, t\right)$ it is not difficult to prove that

$$
\left|g_{2}(t, z)\right| \leqq C_{2}(t, \varepsilon) \rho^{\Re h-1}\left(\gamma_{1}-\rho_{1} \varepsilon\right)^{-N_{1}}, \quad \Re(t)>0,
$$

where $C_{2}(t, \varepsilon)$ is of the same form as $C_{1}(t, \varepsilon)$, used in (3.5).

We substitute (3.9) in (3.4) and we obtain

$$
S_{N_{0}}(z)=(-1)^{N_{0}} \frac{e^{-i \theta\left(a+N_{0}-1\right)}}{\Gamma(a) \Gamma(b)} \sum_{n=0}^{N_{1}-1} b_{n, 1} \int_{0}^{\infty} e^{-\rho \tau} \tau^{a+N_{0}-1}\left(\tau-\gamma_{1}\right)^{n} d \tau+S_{N_{1}}(z),
$$

where

$$
S_{N_{1}}(z)=(-1)^{N_{0}} \frac{e^{-i \theta\left(a+N_{0}-1\right)}}{\Gamma(a) \Gamma(b)} \int_{0}^{\infty} g_{2}(\tau, z) e^{-\rho \tau} \tau^{a+N_{0}-1}\left(\tau-\gamma_{1}\right)^{N_{1}} d \tau .
$$

Again, in [5] it is explained how to compute the integrals in (3.13). With (3.12) we estimate

$$
\left|S_{N_{1}}(z)\right| \leqq C_{2} \rho^{\Re b-1} \int_{0}^{\infty} e^{-\rho \tau} \tau^{\Re a+N_{0}-1}\left|\frac{\tau-\gamma_{1}}{\gamma_{1}-\rho_{1} \varepsilon}\right|^{N_{1}} d \tau,
$$

where $C_{2}$ only depends on $a, b$ and $\varepsilon$. The graph of the integrand of (3.15) looks like Figure 2.2; it has two local maxima, and it is not difficult to prove that the furthest right maximum is the global maximum. This maximum is minimal for $N_{1}=\rho\left(\gamma_{1}-\rho_{1} \varepsilon\right)^{2}\left(2 \gamma_{1}-\rho_{1} \varepsilon\right)^{-1}$. So we choose $N_{1}=\left[\rho\left(\gamma_{1}-\rho_{1} \varepsilon\right)^{2}\left(2 \gamma_{1}-\rho_{1} \varepsilon\right)^{-1}\right]$, and we call $\gamma_{2}$ the place where the integrand has its maximum. We have 
$\gamma_{2}=2 \gamma_{1}-\rho_{1} \varepsilon+\mathcal{O}\left(\rho^{-1}\right), \quad$ as $\rho \rightarrow \infty$, and for small $\varepsilon$ we obtain $\left|S_{N_{1}}(z)\right|=\mathcal{O}\left(\rho^{: * b-\frac{3}{2}} e^{-(1.30 \ldots) \rho}\right)$, as $\rho \rightarrow \infty$.

Next we want to expand $g_{2}(\tau, z)$ in a Taylor series at $\tau=\gamma_{2}$. By writing $g_{2}(\tau, z)=\left\{g_{1}(\tau, z)-\sum_{n=0}^{N_{1}-1} b_{n, 1}\left(\tau-\gamma_{1}\right) n\right\}\left(\tau-\gamma_{1}\right)^{-N_{1}}$, it follows that the Taylor series of $g_{2}(\tau, z)$ at $\tau=\gamma_{2}$ can be computed with the Taylor series of $g_{1}(\tau, z)$ at $\tau=\gamma_{2}$. We write

$$
g_{2}(\tau, z)=b_{0,2}+b_{1,2}\left(\tau-\gamma_{2}\right)+\ldots+b_{N_{2}-1,2}\left(\tau-\gamma_{2}\right)^{N_{2}-1}+\left(\tau-\gamma_{2}\right)^{N_{2}} g_{3}(\tau, z),
$$

and we obtain

$$
\begin{aligned}
S_{N_{1}}(z)=(-1)^{N_{0}} \frac{e^{-i \theta\left(a+N_{0}-1\right)}}{\Gamma(a) \Gamma(b)} & \sum_{n=0}^{N_{2}-1} b_{n, 2} \\
& \times \int_{0}^{\infty} e^{-\rho \tau} \tau^{a+N_{0}-1}\left(\tau-\gamma_{1}\right)^{N_{1}}\left(\tau-\gamma_{2}\right)^{n} d \tau+S_{N_{2}}(z),
\end{aligned}
$$

where

$$
S_{N_{2}}(z)=(-1)^{N_{0}} \frac{e^{-i \theta\left(a+N_{0}-1\right)}}{\Gamma(a) \Gamma(b)} \int_{0}^{\infty} g_{3}(\tau, z) e^{-\rho \tau} \tau^{a+N_{0}-1}\left(\tau-\gamma_{1}\right)^{N_{1}}\left(\tau-\gamma_{2}\right)^{N_{2}} d \tau .
$$

With estimates for $g_{3}(t, z)$, which look like (3.12), we estimate

$$
\left|S_{N_{2}}(z)\right| \leqq C_{3} \rho^{: ß b-1} \int_{0}^{\infty} e^{-\rho \tau} \tau^{\Re i a+N_{0}-1}\left|\frac{\tau-\gamma_{1}}{\gamma_{1}-\rho_{1} \varepsilon}\right|^{N_{1}}\left|\frac{\tau-\gamma_{2}}{\gamma_{2}-\rho_{2} \varepsilon}\right|^{N_{2}} d \tau
$$

Now the integrand has three local maxima, at $\tau=s_{1}, s_{2}, s_{3}$, with $s_{1}<s_{2}<s_{3}$. The local maxima at $s_{1}$ and $s_{2}$ are decreasing functions of $N_{2}$, and the local maximum at $s_{3}$ is decreasing on the interval $s_{3} \in\left(\gamma_{2}, 2 \gamma_{2}-\rho_{2} \varepsilon\right)$, and increasing on the interval $s_{3} \in\left(2 \gamma_{2}-\rho_{2} \varepsilon, \infty\right)$. For small and medium $N_{2}$, the global maximum is at $s_{1}$, but for a certain large $N_{2}\left(N_{2} \sim(1.85 \ldots) \rho\right.$ with $\varepsilon$ small $)$ the global maximum is minimal and it is attained at $s_{1}$ and $s_{3}$. With the aid of a computer we were able to show that $\left|S_{N_{2}}(z)\right|=\mathcal{O}\left(\rho^{\sharp i b-\frac{3}{2}} e^{-(1.94 \ldots) \rho}\right)$, as $\rho \rightarrow \infty$.

An expansion of $g_{3}(\tau, z)$ at $\tau=s_{1}$ will give an estimate of $\left|S_{N_{3}}(z)\right|$, which is of the form (3.19), with an extra factor $\left|\left(\tau-s_{1}\right)\left(s_{1}-\rho_{3} \varepsilon\right)^{-1}\right|^{N_{3}}$ in the integrand. When $N_{3} / \rho$ is bounded away from 0 , for large $\rho$, the extra factor is too large in a neighbourhood of $s_{3}$ and the estimate of $\left|S_{N_{3}}(z)\right|$ explodes. Thus, to obtain the next level of the hyperasymptotic expansion, we expand $g_{3}(\tau, z)$ at $\tau=\gamma_{3}=s_{3}$. The method for obtaining this level is the same as the method for obtaining the previous levels, but many more terms are needed for a large exponential improvement. This follows from the estimate

$$
\begin{aligned}
\left|S_{N_{3}}(z)\right| \leqq C_{4} \rho^{\Re r b-1} \int_{0}^{\infty} e^{-\rho \tau} \tau^{\Re a+N_{0}-1} & \\
& \times\left|\frac{\tau-\gamma_{1}}{\gamma_{1}-\rho_{1} \varepsilon}\right|^{N_{1}}\left|\frac{\tau-\gamma_{2}}{\gamma_{2}-\rho_{2} \varepsilon}\right|^{N_{2}}\left|\frac{\tau-\gamma_{3}}{\gamma_{3}-\rho_{3} \varepsilon}\right|^{N_{3}} d \tau,
\end{aligned}
$$

and now the global maximum of this integrand is greater than the previous 
maximum times $\left[\left(\gamma_{3}-s_{1}\right)\left(\gamma_{3}-\rho_{3} \varepsilon\right)^{-1}\right]^{N_{3}}$. But the method still works and with this method we can obtain $n$ th-level hyperasymptotic expansions.

Note. Olver's expansion (1.5) is obtained by substituting

$$
U(1-b, c, \rho \tau)=(\rho \tau)^{b-1} \sum_{m=0}^{M-1}(-1)^{m} \frac{(1-a)_{m}(1-b)_{m}}{m !(\rho \tau)^{m}}+R_{M}^{\prime}(\rho \tau)
$$

in (3.1). The remainder $R_{M}(z)$ in (1.5) is

$$
R_{M}(z)=\frac{(-1)^{N+M} e^{-i \theta(a+N-1)}}{\Gamma(a) \Gamma(b) \Gamma(1-a) \Gamma(1-b)} \int_{0}^{\infty} \frac{e^{-\rho \tau} \tau^{a+N-1}}{\tau+e^{i \theta}} \int_{0}^{\infty} \frac{U(a, c, \rho \tau s) e^{-\rho \tau s} s^{M-b}}{s+1} d s d \tau,
$$

where $M<N$. This substitution of the asymptotic expansion of the $U$-function in the remainder can be repeated, and a hyperasymptotic expansion is obtained. This method is described in more detail in [2]. In that paper it is proved that at each level the optimal number of terms (the $N_{n}$ ) is smaller than the optimal number of terms at the previous level. The final error in [2] is $R_{N_{x}}(z)=\mathcal{O}\left(e^{-(2.39 \ldots)|z|}\right)$, as $|z| \rightarrow \infty$. In this method, the universal hyperterminants are "horrible" multiple integrands, while the coefficients are very simple. In our method, the universal components are polynomials (see [5]), easily calculated by recursion, but the coefficients, the $b_{n m}$, are more difficult to obtain.

At each level we obtain $\left|S_{N_{n}}(z)\right|=\mathscr{O}\left(|z|^{\mid \Re b-\frac{3}{2}} e^{-\lambda_{n}|z|}\right)$ as $|z| \rightarrow \infty$, where $\lambda_{0}<\lambda_{1}<\lambda_{2}<\ldots$, and $\delta \leqq \arg (z) \leqq 2 \pi-\delta$. For the first five of the $\lambda_{n}$ we have $\lambda_{0} \sim 1, \lambda_{1} \sim 1.30, \lambda_{2} \sim 1.94, \lambda_{3} \sim 2.38$ and $\lambda_{4} \sim 2.46$, as $|z| \rightarrow \infty$. Thus in neighbourhoods of the Stokes lines our $\lambda_{4}$ already exceeds Berry and Howls' limiting value of $2 \cdot 39$.

\section{Discussion}

In Section 2 and in [5], we have shown how a hyperasymptotic expansion can be obtained for $U(a, c, z)$. This expansion is valid far from the Stokes lines. This method can be generalised to any function $F(z)$ expressible as a Laplace transform:

$$
F(z)=\int_{0}^{\infty} t^{a-1} e^{-z t} f(t) d t
$$

where $\Re a>0$, and $f(t)$ is an analytic function in a sector containing $[0, \infty)$, with $|f(t)| \leqq C e^{\sigma t}, t \in[0, \infty), C, \sigma>0$.

In Section 3 it was shown how a hyperasymptotic expansion can be obtained for $U(a, c, z)$, which is valid near the Stokes line. This method can be generalised to any function $G(z)$ expressible as a Stieltjes transform of the form:

$$
G(z)=\int_{0}^{\infty} t^{a-1} e^{-z t} \frac{f(t)}{t+1} d t
$$

with the same conditions on $f$ and $a$.

At present, there are two main methods in the literature for obtaining hyperasymptotic expansions of integrals. In the first method, of Berry and Howls, the "difficult part" of the integral representing the remainder (the function 
TABLE 5.1

Hyperasymptotic approximations to $U\left(\frac{1}{2}, 1, z\right)$ for $z=10 e^{\pi i}$ and $\varepsilon=\frac{1}{1(0)}$

\begin{tabular}{lccl}
\hline Level & Approximation & Exact-approximation & \\
\hline zeroth & $-0.32531887427684 i$ & $1.4 \times 10^{-5}+1.2 \times 10^{-6} i$ & $N_{0}=11$ \\
$U F$ & $0 \cdot 00001403163469-0.32531593185480 i$ & $-1.7 \times 10^{-6} i$ & $N_{1}=5$ \\
first & $0 \cdot 00001403163469-0.32531763374864 i$ & $1.4 \times 10^{-8} i$ & $N_{2}=19$ \\
second & $0 \cdot 00001403163469-0.32531762008956 i$ & $-1.8 \times 10^{-11} i$ & $N_{3}=67$ \\
third & $0 \cdot 00001403163469-0.32531762010763 i$ & $-9.2 \times 10^{-14} i$ & \\
exact & $0 \cdot 00001403163469-0.32531762010773 i$ & 0 & \\
\hline
\end{tabular}

$\tau \mapsto U(1-b, c, \rho \tau)$ in (3.1)), is expanded in a simple asymptotic expansion. The next level of the hyperasymptotic expansion is obtained by substituting the simple asymptotic expansion into the integral representing the remainder. This method is used in $[2,4,8,9,10]$. In [9], Paris starts with a Mellin-Barnes integral representation of $U(a, c, z)$, and he obtains expansion (1.5). The advantage of the first method is that it remains valid across the Stokes lines.

The second method was anticipated in [3]. Boyd indicates how hyperasymptotic expansions of Stieltjes transforms can be obtained. This method is worked out in more detail in [5]. In this paper we extend this method to neighbourhoods of Stokes lines. We start by expanding the "large parameter independent part" of the integral representing the remainder (the function $f_{n+1}(\tau)$ in (2.9)), in a Taylor series at the main saddle point. The next level of the hyperasymptotic expansion is obtained by substituting the Taylor series into the integral representing the remainder. With this method we can achieve greater accuracy.

\section{Numerical illustrations}

We illustrate the method of the previous sections with the $K$-Bessel function. We choose $a=b=\frac{1}{2}$ and have

$$
U\left(\frac{1}{2}, 1, z\right)=\pi^{-\frac{1}{2}} e^{\frac{1}{2} z} K_{0}\left(\frac{1}{2} z\right) .
$$

In the following illustrations we calculate the $U F$-contribution of (3.2), that is the second term on the right-hand side of (3.2), "exact". The zeroth-level is (2.5). The $U F$-level is the zeroth-level plus the $U F$-contribution of (3.2). The first level is the previous plus (3.13). And so on.

Notice that on the Stokes line $\arg (z)=\pi$ the $U F$-contribution of (3.2) takes

TABLE 5.2

Hyperasymptotic approximations to $U\left(\frac{1}{2}, 1, z\right)$ for $z=10 e^{\frac{1}{2} \pi i}$ and $\varepsilon=\frac{1}{100}$

\begin{tabular}{lcccc}
\hline Level & Approximation & Exact-approximation & \\
\hline zeroth & $0 \cdot 22848404036066-0 \cdot 21753754623525 i$ & $6.9 \times 10^{-9}-2 \cdot 4 \times 10^{-6} i$ & $N_{0}=11$ \\
$U F$ & $0 \cdot 22848580197590-0 \cdot 21753930930013 i$ & $-1 \cdot 7 \times 10^{-6}-6.4 \times 10^{-7} i$ & \\
first & $0 \cdot 22848403527347-0 \cdot 21753996260505 i$ & $1 \cdot 2 \times 10^{-8}+8.9 \times 10^{-9} i$ & $N_{1}=5$ \\
second & $0 \cdot 22848404727888-0 \cdot 21753995365582 i$ & $-1.4 \times 10^{-11}-1.3 \times 10^{-11} i$ & $N_{2}=19$ \\
third & $0 \cdot 22848404726479-0.21753995366845 i$ & $-6.9 \times 10^{-14}-6.5 \times 10^{-14} i$ & $N_{3}=67$ \\
exact & $0 \cdot 22848404726472-0 \cdot 21753995366852 i$ & 0 & 0 \\
\hline
\end{tabular}


into account the real part of the $U$-function. This $U F$-contribution is of the same order as $R_{N_{0}}\left(10 e^{\pi i}\right)$, and it is due to this $U F$-contribution that the hyperasymptotic expansion in Section 2 is limited to $|\arg (z)|<\pi$.

In these numerical illustrations the third level approximation is better than the final approximation that can be obtained with the method described at the end of Section 3.

\section{Appendix}

For obtaining estimates for $g_{1}(t, z)$ on $\Re t>0$, we write $z=\rho e^{i \theta}$ and $t=s e^{i v}$, where $0<\theta<2 \pi$ and $-\frac{1}{2} \pi<v<\frac{1}{2} \pi$. We use (1.1) in (3.3) and obtain

$$
\begin{aligned}
g_{1}(t, z)= & \frac{\rho^{b-1}}{\Gamma(1-b)} \int_{0}^{\infty} e^{-y} y^{-b} \\
& \times\left[\frac{\left(1+\frac{y}{\rho s e^{i v}}\right)^{a-1}\left(s e^{i v}\right)^{b-1}-\left(1+\frac{y}{\rho e^{i(\theta-\pi)}}\right)^{a-1}\left(e^{i(\theta-\pi)}\right)^{b-1}}{s e^{i v}-e^{i(\theta-\pi)}}\right] d y,
\end{aligned}
$$

where the function between brackets is bounded for $s>\varepsilon$. Thus

$$
\left|g_{1}(t, z)\right| \leqq C_{1}^{+} \rho^{\Re b-1}, \quad \Re i t>0 \text { and }|t|>\varepsilon .
$$

For $|t|<\varepsilon$, we first estimate $U(1-b, c, \rho t)$ with

$$
|U(1-b, c, \rho t)| \leqq \frac{1}{|\Gamma(1-b)|} \int_{0}^{\infty} e^{-\rho s x} x^{-\Re b b}(1+x)^{\sharp i a-1} d x .
$$

In the case where $\Re i a \leqq 1$, we can estimate the right-hand side of (A.3) with

$$
\begin{aligned}
\frac{1}{|\Gamma(1-b)|} \int_{0}^{\infty} e^{-\rho s x} x^{-\Re h b}(1+x)^{\Re a-1} d x & \leqq \frac{1}{|\Gamma(1-b)|} \int_{0}^{\infty} e^{-\rho s x} x^{-\Re b} d x \\
& \leqq \frac{\Gamma(1-\Re b)}{|\Gamma(1-b)|}(\rho s)^{\Re h b-1}
\end{aligned}
$$

In the case where $\Re i a>1$, we can estimate the right-hand side of (A.3) with

$$
\begin{aligned}
& \frac{1}{|\Gamma(1-b)|} \int_{0}^{\infty} e^{-\rho s x} x^{-\xi h}(1+x)^{\Re a-1} d x=\frac{\rho^{: h-1}}{|\Gamma(1-b)|} \int_{0}^{\infty} e^{-s y} y^{-\aleph b}\left(1+\frac{y}{\rho}\right)^{\Re(a-1} d y \\
& \leqq \frac{\rho^{: i b-1}}{|\Gamma(1-b)|} \int_{0}^{\infty} e^{-s y} y^{-* i b}(1+y)^{\Re i a-1} d y \\
& =\frac{\Gamma(1-\Re b)}{|\Gamma(1-b)|} \rho^{: \Re b-1} U(1-\Re b, \Re i c, s) \\
& \varliminf_{*} C \rho^{: i b-1} s^{-|: i c|} \text {. }
\end{aligned}
$$

In $*$ we used that $U(\alpha, \gamma, z)=o\left(z^{-|:| \gamma \gamma \mid}\right)$, as $z \rightarrow 0$. This rough estimate follows from $[1,13.5 .5 \ldots 13.5 .12]$. Thus we obtain the estimate

$$
|U(1-b, c, \rho t)| \leqq C^{\Re \mid r-1} s^{-|\Re| i|-| \Re|r|,},
$$


and finally

$$
\left|g_{1}(t, z)\right| \leqq C_{1}^{-} \rho^{\Re i b-1}|t|^{-|\Re a|-|\Re b|}, \quad \Re t>0 \text { and }|t| \leqq \varepsilon .
$$

Estimate (3.5) is the union of estimates (A.2) and (A.7).

For the proof of (3.7), we start with

$$
\begin{aligned}
g_{1}(t, z) & =\frac{1}{\Gamma(1-b)} \int_{0}^{\infty} \frac{e^{-\rho t x}-e^{\rho e^{i \theta} x}}{t+e^{i \theta}} x^{-b}(1+x)^{a-1} d x \\
& =-\frac{\rho}{\Gamma(1-b)} \int_{0}^{\infty} \int_{0}^{1} e^{\rho x y\left(t+e^{i \theta}\right)} d y e^{-\rho t x} x^{1-b}(1+x)^{a-1} d x \\
& =-\rho \frac{\Gamma(2-b)}{\Gamma(1-b)} \int_{0}^{1} U\left(2-b, c+1, \rho\left[t(1-y)-y e^{i \theta}\right]\right) d y
\end{aligned}
$$

In the following derivation, we use $\left(d^{n} / d y^{n}\right) U(\alpha, \gamma, y)=(-y)^{n}(\alpha)_{n}$ $U(\alpha+n, \gamma+n, y)$

$$
\begin{aligned}
\frac{d^{n}}{d t^{n}} g_{1}(t, z)= & -\rho(1-b) \int_{0}^{1} \frac{d^{n}}{d t^{n}} U\left(2-b, c+1, \rho\left[t(1-y)-y e^{i \theta}\right]\right) d y \\
= & (-\rho)^{n+1}(1-b)_{n+1} \int_{0}^{1}(1-y)^{n} \\
& \times U\left(2-b+n, c+n+1, \rho\left[t(1-y)-y e^{i \theta}\right]\right) d y \\
= & \frac{(-\rho)^{n}(1-b)_{n}}{t+e^{i \theta}}[U(1-b+n, c+n, \rho t) \\
& \left.-n \int_{0}^{1}(1-y)^{n-1} U\left(1-b+n, c+n, \rho\left[t(1-y)-y e^{i \theta}\right]\right) d y\right] \\
= & \frac{1}{t+e^{i \theta}}\left[(-\rho)^{n}(1-b)_{n} U(1-b+n, c+n, \rho t)-n \frac{d^{n-1}}{d t^{n-1}} g_{1}(t, z)\right] .
\end{aligned}
$$

\section{References}

1 M. Abramowitz and I. A. Stegun. Handbook of mathematical functions with formulas, graphs and mathematical tables. Nat. Bur. Standards Appl. Series 55 (Washington, D.C.: U.S. Government Printing Office, 1964).

2 M. V. Berry and C. J. Howls. Hyperasymptotics for integrals with saddles. Proc. R. Soc. London Ser. A. 434 (1991), 657-675.

3 W. G. C. Boyd. Stieltjes transform and the Stokes phenomenon. Proc. R. Soc. London Ser. A. 429 (1990), 227-246.

4 D. S. Jones. Uniform asymptotic remainders. In Asymptotic and Computational Analysis, ed. R. Wong, pp. 477-90 (New York: Marcel Dekker, 1990)

5 A. B. Olde Daalhuis. Hyperasymptotic expansions of confluent hypergeometric functions. IMA J. Appl. Math. 49 (1992), 203-216.

6 F. W. J. Olver. Asymptotics and Special Functions (London/New York: Academic Press, 1974).

7 F. W. J. Olver. Uniform, exponentially improved, asymptotic expansions for the generalized exponential integral. SIAM J. Math. Anal. 22 (1991), 1460-1474.

8 F. W. J. Olver. Uniform, exponentially improved, asymptotic expansions for the confluent hypergeometric function and other integral transforms. SIAM J. Math. Anal. 22 (1991), $1475-1489$. 
9 R. B. Paris. Smoothing of the Stokes phenomenon using Mellin-Barnes integrals. J. Comp. Appl. Math. 41 (1992), 117-133.

10 R. B. Paris. Smoothing of the Stokes phenomenon for high-order differential equations. Proc. $R$. Soc. London Ser. A. 436 (1992), 165-186.

(Issued 11 August 1993) 